\title{
As práticas do cuidar na oncologia: a experiência da fisioterapia em pacientes com câncer de mama
}

\section{Care practice in oncology: the physical therapy experience with breast cancer patients}

Lina Faria

Pesquisadora do Instituto de Medicina Social/ Universidade Estadual do Rio de Janeiro

Condomínio Village, casa 19

22202-317 - Petrópolis - RJ - Brasil lina@compuland.com.br

Recebido para publicação em outubro de 2008. Aprovado para publicação em abril de 2009.
FARIA, Lina. As práticas do cuidar na oncologia: a experiência da fisioterapia em pacientes com câncer de mama. História, Ciências, Saúde - Manguinhos, Rio de Janeiro, v.17, supl.1, jul. 2010, p.69-87

\section{Resumo}

A fisioterapia oncológica precoce vem desempenhando um importante papel na prevenção e minimização dos efeitos adversos do tratamento do câncer de mama, que acomete um grande número de mulheres. A fisioterapia reduz os riscos de complicações e pode restaurar a integridade cinético-funcional de órgãos e sistemas. A fisioterapia oncológica, ao enfatizar os caminhos da prevenção, tem ampliado a atuação do fisioterapeuta e consolidado seu espaço legítimo no campo médico, complementando as habilidades e competências adquiridas nos últimos anos. A prevenção de problemas e a promoção da saúde estão hoje entre as principais atribuições do fisioterapeuta e devem estar presentes em todas as fases do câncer de mama, do diagnóstico ao tratamento e aos cuidados paliativos.

Palavras-chave: fisioterapia oncológica precoce; câncer de mama; atuação profissional.

\section{Abstract}

Early oncology physical therapy has played a vital role in preventing and minimizing theside effects of treatment for breast cancer, a disease that strikes a large number of women. Physical therapy reduces the risks of complications and can restore the kinetic and functional integrity of organs and systems. W ith this emphasis on means of prevention, oncology physical therapy has expanded the professional activities of physical therapists and added to their required skill set in recent years, thereby reinforcing the field's legitimatespace within medicine. One of a physical therapist's main duties today is to prevent problems and promote good health, and this service should be offered during all phases of breast cancer, from diagnosis through treatment and palliativecare.

Keywords: early oncology physical therapy; breast cancer; professional activities. 
A fisioterapia é uma ciência aplicada, cujo objeto de estudo é "o movimento humano em todas as suas formas de expressão", quer nas alterações patológicas, psíquicas ou orgânicas (Brasil, 21 maio 1987). Mesmo em outros tempos, quando essa área do saber ainda se subordinava aos ditames da profissão médica, seu campo de ação já se delineava nos maiores centros científicos do mundo. O Consel ho de Fisioterapia da Associação Médica Americana, em 1937, assim a definia: “É um termo empregado [no] tratamento da enfermidade por vários meios físicos, compreendendo o emprego do calor, da massagem, do exercício, da luz, da água, da el etricidade e el ementos mecânicos e físicos de outros gêneros" (Asociación Médica Americana, 1937). ${ }^{1}$

Desde os primeiros tempos de sua formação, a atividade fisioterapêutica volta-se para os processos de recuperação do indivíduo por meio de um conjunto de técnicas corporais que agem sobre o organismo humano, imprimindo-Ihe uma mobilização ativa ou passiva, restaurando o gesto e a função das diferentes partes do corpo. Tem como obj etivos principais prevenir, manter e restaurar a integridade dos movimentos, órgãos, sistemas e funções. Trata-se de manter o movimento, segundo Kisner e Colby $(2005$, p.3), sem a ocorrência de sintomas, durante atividades funcionais básicas ou complexas.

É amplo o campo de atuação da fisioterapia nas áreas de ortopedia, neurologia, cardiologia, angiologia, reumatologia, pediatria, ginecologia, geriatria, pneumologia e oncologia. Para cada área apresenta seus recursos de tratamento. A fisioterapia se utiliza de métodos e técnicas que atuam tanto na melhora da sintomatologia quanto na prevenção e qualidade de vida. Os recursos terapêuticos incluem a eletroterapia, a mecanoterapia, a fototerapia e a cinesioterapia. Atua em diferentes disfunções dos sistemas neurolocomotor, musculoesquelético, cardiorrespiratório. ${ }^{2}$

Por ser um profissional que detém conhecimentos amplos, o fisioterapeuta pode atuar em diversos campos da atenção à saúde. $\mathrm{Na}$ clínica realiza diagnóstico cinético-funcional, prognóstico, prescrição, indução do tratamento, reavaliação e alta do paciente. O fisiote rapeuta pode, também, desempenhar suas atividades em ambulatórios, consultórios, centros de reabilitação, áreas que envolvam a saúde coletiva e no ensino (Brasil, 21 maio 1987).

O tratamento fisioterápico é imprescindível para qualquer indivíduo cuja atividade diária esteja comprometida. Nos processos de doença, contribui na redução de quadros dolorosos e evita possíveis complicações após cirurgias ou longos períodos de imobilizações (Kisner, Colby, 2005, p.4).

É importante lembrar que até bem pouco tempo as atribuições dos fisioterapeutas e dos profissionais de saúde, de um modo geral, estavam bastante restritas à doença. As possibilidades de intervenção se voltavam basicamente para o tratamento de patologias e a reabilitação de organismos lesados por meio da terapia. O modelo de saúde era o reabilitador. A prevenção de problemas e a promoção de saúde não faziam parte do leque de atuação do profissional. Havia, segundo Rebelatto e Botomé (1999, p.18-19), uma exclusiva atenção ao tratamento de pessoas fisicamente lesadas.

Ao longo de sua história, portanto, a fisioterapia teve suas ações concentradas em dois níveis de atenção à saúde: o responsável pelo diagnóstico e tratamento das doenças e o responsável pela reabilitação. Isso se deveu, em parte, à própria trajetória da fisioterapia. No Brasil, as epidemias de poliomielite e os al tos índices de acidentes de trabalho, nos anos 
1950, orientaram a atuação profissional para as atividades recuperativas (Rebelatto, Botomé, 1999, p.20; Barros, 2008).

Além disso, a ausência de uma tradição em ensino e pesquisa, de cursos para formação de profissionais e a pouca experiência em instituições de saúde contribuíram para ratificar essa situação. Mesmo quando da criação dos primeiros cursos no país, os currículos continuavam a intensificar o ensino de técnicas e métodos de trabal ho. Poucos visavam uma atuação profissional socialmente significativa, transformadora da realidade da saúde brasileira. A mudança de orientação foi um processo lento e gradativo.

Aos poucos, as atividades curativas e reabilitadoras foram cedendo espaço à atenção primária em saúde, ampliando significativamente o leque de intervenção da fisioterapia. Novas especialidades foram sendo criadas, privilegiando a área da prevenção e promoção. É possível dizer que o surgimento da fisioterapia oncológica acompanhou esse processo de mudança da intervenção fisioterapêutica.

A fisioterapia em oncologia é uma especialidade recente e tem como metas preservar e restaurar a integridade cinético-funcional de órgãos e sistemas, assim como prevenir os distúrbios causados pelo tratamento oncológico. No Instituto Nacional do Câncer (Inca), no Rio de Janeiro, teve seu início nos anos 1980, vinculada ao Serviço de Cirurgia Torácica. A fisioterapia oncológica lida com as sequelas próprias do tratamento, atuando de forma preventiva para minimizá-las. ${ }^{3}$

Em relação aos pacientes que passaram por uma mastectomia de mama, a fisioterapia oncológica atua no pós e no pré-operatório. Aborda vários aspectos, desde a preparação do paciente para a cirurgia até a reabilitação funcional no pós-cirúrgico. $O$ paciente que se submete a um programa preventivo no préoperatório diminui o tempo de internação e retorna mais rapidamente às atividades diárias e ocupacionais. Num contexto mais amplo, um programa fisioterapêutico preventivo auxilia evitando complicações comuns no paciente mastectomizado (Bergmann, 2008).

\section{A formação do campo da fisioterapia no Brasil: breve histórico}

A institucionalização da fisioterapia como área de ensino e de pesquisa e o estabelecimento da comunidade de fisioterapeutas no Brasil ainda são processos recentes. Descrever o modo pelo qual a consolidação da comunidade e o amplo campo de atuação da fisioterapia se processaram no país não é uma tarefa muito fácil. Essa dificuldade está relacionada ao estudo da emergência e consolidação de qualquer profissão, em especial aquelas que nasceram como uma especialização da área médica. Vejamos, por exemplo, o caso da enfermagem de saúde pública.

A enfermagem, a duras penas, conquistou um espaço que desafiava a hegemonia médica, particularmente no trabalho de saúde pública (Castro Santos, 2008). No cenário brasileiro, desde o final da década de 1920 a enfermeira foi cada vez mais reconhecida como um ator importante dos serviços de saúde pública. Nos centros de saúde e no trabalho de visitação às famílias, a enfermagem se legitimava como profissão. A base de seu trabal ho estava na educação sanitária, levada às famílias em centros urbanos e nos trabalhos de profilaxia rural (Castro Santos, Faria, 2005; Faria, 2006; Castro Santos, Faria, 2008). 
Segundo Carrara (1996, p.75), a emergência e consolidação de qualquer especialidade realizam-se simultaneamente em vários planos. É preciso considerar, em primeiro lugar, o momento em que a comunidade científica se estabelece, com seus congressos, sociedade, centros de pesquisa, periódicos e fontes de financiamento. Em segundo, é importante a instituição do ensino especializado, com suas cátedras, concursos, teses e, ainda, no caso de uma ciência aplicada, como é a fisioterapia, a abertura e expansão de um mercado de novos serviços.

No final do século XIX e início do XX novos centros de pesquisa, institutos, faculdades, universidades e laboratórios foram criados, em resposta ao movimento da comunidade científica brasileira no sentido de valorização e expansão do trabal ho científico. Nesse período, começaram a surgir as várias especializações na área da medicina e cursos para formação de profissionais na área da saúde. Essa implantação se realizou entre as duas últimas décadas do século XIX e primeiras três décadas do século XX. Essas instituições passam a ser vistas como um local para formação de novos pesquisadores, imbuídos de um padrão de trabalho científico (Schwartzman, 1979; Santos Filho, 1980, 1991).

Alguns marcos fundamentais na história institucional da fisioterapia no Brasil e em outros países podem ser apontados. Segundo Figueirôa (1996), foi logo após a Primeira Guerra Mundial que começaram a surgir os grandes centros de reabilitação, de reaprendizagem do movimento e reeducação funcional. Na Alemanha, por exemplo, as primeiras escolas foram as de Kiel (1902) e Dresden (1918). De acordo com Sanchez, foram muitas as parcerias entre fisioterapeutas e médicos nessa época, destacando-se o exemplo do médicocirurgião alemão Rudolf Klapp, que em parceria com as fisioterapeutas Blederbeck e Hess desenvolveram o Método Klapp. Na Inglaterra, a parceria entre a fisioterapeuta Berta Bobath e o neurofisiologista Karel Bobath foi uma das mais frutíferas (Sanchez, 1984, p.29). ${ }^{4}$

Nos EUA, a primeira associação de fisioterapia foi criada em 1921 - American Physical Therapy Association. A partir de então, vários departamentos de fisioterapia foram organizados nos diversos hospitais americanos. Essa teria sido, de acordo com o Consel ho de Fisioterapia da Associação M édica Americana, uma solução condizente com o desenvolvimento de uma "legítima prática fisioterápica" (Asociación Médica Americana, 1937, p.318). ${ }^{5}$

Em 1951 foi fundada, em Londres, a World Confederation for Physichal Therapy (WCPT), com a adesão de 13 países. Em 2001 a WCPT representava mais de 82 paísesmembros, inclusive o Brasil, congregan do mais de 225 mil fisioterapeutas em todo o mundo (Barros, 2003).

Na América Latina, as primeiras instituições foram organizadas entre as décadas de 1920 e 1950. A crescente urbanização e industrialização, o surgimento das epidemias de poliomielite e os primeiros centros de reabilitação criaram novas possibilidades para os fisioterapeutas. Na Argentina, o Instituto Municipal de Radiologia e Fisioterapia de Buenos Aires, fundado em 1925, tinha como propósito prestar serviços especializados e formação profissional em fisioterapia (Opas, 1941). Na Colômbia, em 1952, foi criada a Escola Nacional de Fisioterapia, e em 1956, no Chile, foi organizada a Escola de Fisioterapia da Universidade do Chile (Barros, 2008, p.946). 
No cenário brasileiro, em relação ao ensino, São Paulo tomaria a dianteira. Ainda em 1929 foi criado o Serviço de Fisioterapia do Instituto do Radium Arnal do Vieira deCarval ho ${ }^{6}$, para dar assistência aos pacientes do Hospital Central da Santa Casa de Misericórdia, sob a direção de Ovídio Pires de Campos. O Instituto do Radium surgiu do empenho de Vieira de Carval ho ao sugerir a criação de um centro de radioterapia, em razão do número crescente de casos de câncer em São Paulo. O Instituto se caracterizava por ser uma instituição filantrópica sem fins lucrativos, cujo objetivo era o diagnóstico, a preven ção e o tratamento do câncer (Rebelatto, Botomé, 1999, p.49; Marques, Sanches, 1994, p.5-6).7 Nesse mesmo ano foi organizado o Serviço de Fisioterapia do Hospital das Clínicas, iniciando-se, em 1951, o primeiro curso para a formação de técnicos em fisioterapia no país. A meta era formar técnicos para as atividades predominantemente curativas e reabilitadoras (Sanchez, 1984, p.31). ${ }^{8}$ Segundo Oliveira (2004, p.249), os primeiros cursos foram organizados e ministrados basicamente por médicos e enfermeiras, que não tinham claro quais disciplinas deveriam ser oferecidas aos alunos.

Algum tempo depois, Godoy Moreira, professor da Faculdade de Medicina da Universidade de São Paulo (USP), fundou, em conjunto com a Organização Pan-Americana da Saúde/Organização Mundial da Saúde (Opas/OMS) e a WCPT, o Instituto Nacional de Reabilitação, anexo à cadeira de Ortopedia e Traumatologia daquela faculdade. Um novo curso para formação de técnicos em fisioterapia foi organizado, com duração de dois anos, para atender aos programas de reabilitação que a Opas estava interessada em desenvolver na América Latina. Algum tempo depois, a Reitoria da USP regulamentou o curso (Marques, Sanchez, 1994, p.6). ${ }^{9}$

Enquanto isso, no Rio de Janeiro, em 1954, estava sendo fundada a Associação Brasileira Beneficente de Reabilitação (ABBR), o primeiro centro no país voltado para a paralisia infantil, cujo idealizador foi o arquiteto Fernando Lemos. Dois anos depois, foi criado, por Juscelino Kubitscheck, o Centro de Reabilitação na Associação Beneficente, e em 1956 começaram a funcionar os cursos para formação de fisioterapeutas e terapeutas ocupacionais (Batista, 1988) na Escola de Reabilitação do Rio de Janeiro (ERRJ), a primeira instituição no país a oferecer um curso regular de graduação em fisioterapia (Brasil , 2 dez. 1965). A formação dos fisioterapeutas acontecia em salas de aula da própria ABBR. Os alunos também se deslocavam para outras instituições, como a Policlínica do Rio de Janeiro, Hospital Carlos Chagas, Hospital Estadual Getúlio Vargas, Hospital Jesus, Hospital Pedro Ernesto e Cruz Vermelha (Figueirôa, 1996; Reis, 2004).

As disciplinas oferecidas aos alunos eram: anatomia descritiva, fisiologia humana, cine siologia clínica, massoterapia, termoterapia, patologia médica, clínica neurológica, fisiologia humana, cinesioterapia, clínica reumatológica, clínica psicossomática, fundamentos da reabilitação, clínica, ortopédica e clínica traumatológica. Um segundo curso, iniciado em 1957, teve sua duração ampliada para três anos. O último ano era dedicado ao estágio obrigatório, que acontecia no Centro de Reabilitação. Entre 1958 e 1980 foram cerca de quinhentos fisioterapeutas formados nos cursos da ABBR (Barros, 2008, p.948). ${ }^{10}$ Aos poucos esses profissionais foram assumindo as disciplinas nos cursos oferecidos tanto no Rio de Janeiro quanto em outros estados brasileiros. ${ }^{11}$ 
No Hospital das Clínicas de São Paulo começava a funcionar a Clínica Ortopédica e Traumatológica da Faculdade de Medicina de São Paulo, que tinha como finalidade o atendimento aos traumatizados do aparelho locomotor e aos doentes da paralisia infantil. A Clínica era procurada por pacientes de todos os estados do Brasil e do exterior. No Hospital das Clínicas também funcionava o Instituto Nacional de Reabilitação (Inar), implantado pelo decreto 27.083 de 21 de dezembro de 1956, com a finalidade de dar atendimento e recuperação aos operários acidentados. Ambos possuíam serviços de fisioterapia (USP, 1997).

Em 1957 nascia, em São Paulo, a Associação Paulista de Fisioterapeutas (APF) e em agosto de 1959 foi fundada a Associação dos Fisioterapistas do Estado de São Paulo, hoje denominada Associação Brasileira de Fisioterapia (ABF). Essa Associação desempenhou um importante papel na transformação do curso de nível técnico para nível superior, contribuindo para o reconhecimento do fisioterapeuta como profissional de nível superior (Sanchez, 1984, p.8). Nesse período também começaram a ser formuladas leis que regulamentariam a atuação dos fisioterapeutas no Brasil. Muitas vozes levantam-se em defesa da emancipação e da profissional ização da fisioterapia e contra a condição reabilitadora de sua atuação. Editoriais da recém-criada Revista da Associação Brasileira de Fisioterapia passam a criticar as intromissões dos médicos e a reivindicar o direito à soberania profissional, defendendo a capacidade dos próprios profissionais em traçar os rumos da profissão (Barros, 2003; Oliveira, 2004, 2005)..$^{12}$

Em 1962, o Consel ho Federal de Educação estabeleceu o currículo mínimo para a formação universitária em fisioterapia no país. ${ }^{13}$ No entanto, insistia na caracterização desses profissionais como "auxiliares médicos" ou "técnicos em fisioterapia", a desempenhar tarefas de caráter terapêutico sob a orientação e a responsabilidade do médico. Ao médico caberia diagnosticar a doença e orientar a equipe. Ao técnico em fisioterapia caberia apenas executar as técnicas que conduzem à reabilitação do paciente.

No Primeiro Congresso Brasileiro de Fisioterapia, realizado no Rio de Janeiro, entre 12 e 14 de novembro de 1964, o conflito profissional ganhou nova dimensão. A ABF começou a apregoar a autodeterminação como forma de garantir o crescimento e a evolução da profissão de fisioterapeuta no país (Galvão, 2000). ${ }^{14} \mathrm{Em}$ várias capitais brasileiras foram surgindo associações regionais de fisioterapia, ligadas à Associação. Novas escolas foram criadas, no entanto, de acordo com Galvão (2000) eram grandes as resistências por parte dos médicos à consolidação da profissão. Apesar das dificuldades enfrentadas, a ABF procurou centrar suas ações no reconhecimento oficial da profissão.

No final dos anos 1960 a profissão foi regulamentada no país. O decreto-lei 938 assegurava o exercício das profissões de fisioterapeuta e terapeuta ocupacional. Reconhecia esses profissionais como diplomados por escolas e cursos reconhecidos de nível superior, porém definia como atividade executar técnicas fisioterápicas para restaurar e conservar a capacidade física do paciente. Esses profissionais poderiam, ainda, dirigir ou assessorar serviços em estabelecimentos públicos ou particulares, exercer o magistério e supervisionar alunos em atividades práticas (Brasil, 13 out. 1969).

A lei 6.316, decretada em 1975 pelo Congresso Nacional e sancionada pelo presidente Geisel, criou o Conselho Federal de Fisioterapia (Coffito) e o Conselho Regional de 
Fisioterapia e Terapia Ocupacional (Crefito). ${ }^{15} \mathrm{O}$ artigo 13 deixava claro que o fisioterapeuta poderia desempenhar atividades várias em hospitais, clínicas, ambulatórios, asilos, por meio da apresentação de sua carteira profissional de fisioterapeuta.

Embora os avanços tenham sido muitos desde a criação dos primeiros cursos, é importante ressaltar que, ainda hoje, são grandes as limitações que a legislação impõe ao profissional de fisioterapia. Para Salmória e Camargo (2008), dois fatores importantes contribuem para as dificuldades no estabelecimento da profissão, no que se refere ao seu reconhecimento pelos demais profissionais de saúde: o fato de a fisioterapia ainda ser uma profissão recente e a forte presença de profissionais fisioterapeutas jovens no mercado de trabalho.

\section{O câncer e a mastologia}

Ao longo da história das doenças, o câncer foi visto de diversas formas: como uma doença incurável, como uma doença contagiosa, como um problema social, como um problema desaúde pública. Nos meios médicos pouco se sabia sobrecomo evitar o sofrimento e os índices de mortal idade. Em fins do século XIX e inícios do século XX, o câncer começou a ser visto como uma 'enfermidade' merecedora de atenção médico-social, de busca e acumulação de conhecimentos científicos e de medidas de prevenção. As estatísticas de óbitos, que revelaram uma situação crítica, contribuíram para uma tomada de posição por parte de governos e de autoridades da saúde. ${ }^{16}$

O desenvolvimento de novas técnicas de assepsia e antissepsia e os avanços nas cirurgias ampliaram as possibilidades de intervenção. A utilização da radioterapia no tratamento das displasias foi um instrumento importante da medicina contra o câncer. As inovações no tratamento do câncer favoreceram o interesse pela doença nos círculos médicos em vários países, inclusive no Brasil. O câncer passou a ser objeto de pesquisa. As discussões sobre as causas, incidência e possível contagiosidade ganharam espaço nos congressos internacionais de medicina (Teixeira, Fonseca, 2007, p.13-22).

Nos círculos médicos havia a preocupação em conhecer mais sobre o câncer e as novas técnicas de tratamento. Ao contrário de doenças como hanseníase, sífilis e tuberculose, o câncer não era controlado por medidas higiênicas, sendo as cirurgias e a radioterapia as únicas ações profiláticas (Sanglard, 2008, p.189).

A partir de então, começaram a surgir as primeiras instituições de pesquisa, centros de radioterapia e de tratamento especializado na Europa, nos Estados Unidos e no Japão. No Brasil, desde 1920, o Departamento Nacional de Saúde Pública, no âmbito do Ministério da Justiça e Negócios Interiores, abrigava uma Inspetoria de Profilaxia da Lepra, das Doenças Venéreas e do Câncer, primeiro órgão federal destinado à campanha contra a doença no país.

Segundo Mirra (2005, p.1-2), a preocupação em conhecer a incidência do câncer no Brasil era muito antiga. Em 1904 Azevedo Sodré publicou um artigo sobre a frequência da doença no país, na revista Brasil Médico. Ainda segundo o autor, as primeiras publicações oficiais referentes às taxas de mortalidade por câncer datam de 1944, organizadas pelo então Ministério de Educação e Saúde, contendo dados referentes ao período de 1929 a 1932 (Sodré, 1904). Nas primeiras décadas do século XX, os inquéritos epidemiológicos 
realizados nos vários estados brasileiros mostravam que a extensão da doença era ainda maior do que se imaginava.

Os médicos brasileiros não ficaram alheios a esse novo campo de estudos que se inaugurava. Em 1922 foi criado, em Belo Horizonte, o Instituto do Radium, voltado para pesquisa e tratamento do câncer, nos moldes do Instituto de Radium de Paris (Teixeira, Fonseca, 2007, p.34). Algum tempo depois foi inaugurado o Instituto Nacional do Câncer (Inca), responsável pela formulação e implantação da política de atenção oncológica no país, incluindo prestação de serviços médicos, ensino, pesquisa e elaboração de programas educativos. Essas iniciativas podem ser consideradas o início da organização da luta contra o câncer no Brasil.

Devido à incidência das afecções mamárias, uterinas, cervicais, cutâneas, novos serviços foram criados. Tornou-se imprescindível a criação de áreas especializadas. No Inca, desde final dos anos 1930 vinham sendo organizadas sessões especializadas. Em 1957 foi criada a Seção de Mastologia, sob a chefia do médico Alberto Lima de Moraes Coutinho, que a dirigiu entre 1957 e 1967. Alberto Lima foi o pioneiro da mastologia brasileira. Com o intuito de divulgar pesquisas nessa especialidade, organizou vários cursos no Rio de Janeiro e em outros estados brasileiros. Em 1959 fundou a Sociedade Brasileira de Patologia Mamária, hoje Sociedade Brasileira de Mastologia (SBM), da qual foi presidente por três vezes. A atuação de Lima de Moraes foi importante para o reconhecimento da mastologia como especialidade médica (Guimarães et al., 2002, p.135-138).

Atualmente as afecções da mama são consideradas um importante problema de saúde pública no Brasil. ${ }^{17} \mathrm{O}$ carcinoma mamário é a neoplasia mais frequente entre as mulheres e corresponde a uma das principais causas de óbitos no país. Em diversas partes do mundo, e também no Brasil, as campanhas apoiam-se nas orientações médicas de que o câncer é curável se detectado e tratado quando de seu início. Na população brasileira, no entanto, a maioria dos diagnósticos de câncer de mama é realizada em estádios mais avançados, quando se faz necessário instituir tratamentos mais radicais, com aumento significativo da morbidade (Abreu, Koifman, 2002, p.114; Bergmann et al., 2004, p.312).

Os tratamentos radicais incluem a mastectomia, com retirada da mama, dos músculos do peito, de todos os gânglios linfáticos da axila, de al guma gordura em excesso e pele. $O$ papel da fisioterapia oncológica é fundamental na reabilitação do paciente e na prevenção de possíveis complicações.

A fisioterapia oncológica é uma área ainda nova, mas em expansão. No Inca foi organizada em 1980, vinculada ao Serviço de Cirurgia Torácica do Hospital do Câncer I (HC I). Na época contava com poucos fisioterapeutas. No final da década de 1980 e início da seguinte houve a necessidade da incorporação de novos profissionais. Em virtude da demanda crescente de atendimento hospitalar, o espaço físico foi reestruturado em um setor bem equipado e com autonomia de condutas e rotinas de atendimentos..$^{18}$

\section{A fisioterapia em oncologia}

A fisioterapia em oncologia éuma especialidade quetem como objetivo preservar, manter, desenvolver e restaurar a integridade cinético-funcional de órgãos e sistemas, assim como 
prevenir os distúrbios causados pelo tratamento oncológico. ${ }^{19} \mathrm{Em}$ maio de 2008 realizou-se o Terceiro Congresso Brasileiro de Fisioterapia em Cancerologia, voltado exclusivamente para acadêmicos e profissionais da área. Foram discutidos temas relacionados à abordagem fisioterapêutica em pacientes amputados; em pacientes que sofreram cirurgias toracoabdominais; nos cuidados paliativos oncológicos; no ensino e na pesquisa em oncologia; nas políticas públicas de saúde; na fisioterapia respiratória em oncologia; na neurocirurgia oncológica; na mastologia; na oncologia clínica; e na urologia oncológica. Cada vez mais cresce a participação da fisioterapia nessa área, em especial no pré e pós-operatório de câncer de mama, como será mostrado adiante.

\section{As pesquisas em centros mundiais de referência}

A Escola de Saúde Pública de Harvard, reconhecida como uma referência internacional sobre pesquisas de câncer de mama, aponta o extenso lobby das doentes, bem como o impacto da mídia, que geram um intenso debate sobre prevenção, métodos e possibilidades de cuidado e cura. Um importante estudo brasileiro indica, nessa mesma direção, a organização eficiente das mulheres com câncer de mama, que se destacam dos pacientes de câncer prostático justamente pelo grau de organização de seus interesses e pela maior pressão sobre órgãos e recursos públicos e privados (Coutinho, Soares, 2000). Uma matéria sobre as pesquisas realizadas em Harvard confirma o papel crescente do câncer feminino na agenda científica daquele importante centro mundial. O que hoje se sabe sobre os fatores de risco do câncer de mama - em relação à idade da primeira gestação - na verdade resultou de estudos de forte impacto internacional, realizados há mais de trinta anos sob a liderança do epidemiologista e professor da Harvard School of Public Health (HSPH), Brian MacMahon (Pollack, 1992, p.4-10).

A partir de estudos pioneiros como os da equipe de MacMahon, hoje se sabe que os fatores de risco são multifatoriais: fatores hormonais; alterações genéticas; história familiar; menopausa tardia; menarca precoce; primeira gravidez após trinta anos; ingestão regular de álcool; exposição a radiações ionizantes em idade inferior a 35 anos; e nuliparidade (Pollack, 1992, p.4-6). O conhecimento gerado pelas pesquisas, nas últimas décadas, teve impacto direto sobre a prevenção do câncer de mama e al guns resultados que contrariavam hipóteses ou 'achados' até certo ponto consensuais. Por exemplo, a amamentação, associada positivamente à saúde neonatal, não parecia conferir proteção para o câncer de mama. Tampouco se confirmou a hipótese da associação entre obesidade, constituição corporal e risco de câncer de mama na pós-menopausa.

Outros estudos conduzidos em Boston, pelos pesquisadores dos departamentos de Epidemiologia e Nutrição da HSPH, vieram a confirmar hipóteses correntes sobre a associação entre menstruação precoce, menopausa tardia e carcinoma mamário (Pollack, 1992). A mulher ficaria exposta por mais tempo aos altos níveis de estrogênio do corpo. Se por um lado, diziam os estudos, ela estaria protegida das doenças coronarianas e da osteoporose, por outro teria um risco de $40 \%$ de desenvolver a doença.

O papel dos anticoncepcionais na etiologia do câncer de mama também foi bastante discutido na mesma época, quando os resultados de pesquisas apontavam para a elevação 
Lina Faria

do risco de carcinoma pelo consumo de contraceptivos, antes do ciclo reprodutivo feminino. ${ }^{20}$

\section{A fisioterapia e o câncer de mama}

O câncer de mama causa alterações físicas e emocionais importantes nas mulheres. A OMS ressalta que a detecção precoce é fundamental, pois o tratamento é mais efetivo quando a doença é diagnosticada em fases iniciais, antes do aparecimento dos sintomas clínicos (Brasil, 2004, p.84; Brasil, s.d. - Inca, Relatório anual 2005, p.20). Segundo Bergmann (2000), quanto mais precoce o diagnóstico, melhores são as condições de tratamento e maior é a sobrevida das mulheres acometidas.

O câncer de mama é uma doença complexa, com formas de evolução lenta ou rapidamente progressiva. É uma doença sistêmica, envolve vários órgãos. O carcinoma mamário consiste na formação de um tumor maligno a partir da multiplicação acelerada de céulas anormais, podendo apresentar-se através de inúmeras formas clínicas e morfológicas (Guia..., s.d.). É relativamente raro antes dos 35 anos de idade, mas acima dessa faixa sua incidência cresce rápida e progressivamente. O câncer de mama tem cura quando detectado no início. O índice de sucesso no tratamento é de $90 \%$, e quase sempre não é preciso retirar toda a mama (Brasil, 2004, p.84; Brasil, s.d. - Inca, Relatório anual 2007).

O principal sintoma éo nódulo no seio, acompanhado ou não de dor mamária (detectado através do autoexame, do exame feito pelo médico ou por mamografia). Podem surgir alterações na pele que recobre a mama, como abaulamentos ou retrações ou, ainda, nódulos pal páveis na axila e secreção mamilar. ${ }^{21} \mathrm{O}$ rastreamento é feito por meio da mamografia, que permite a detecção precoce, por sua capacidade de local izar lesões em fase inicial (Brasil, s.d.; Brasil, 2004, p.84; Brasil, s.d. - Inca, Relatório anual 2004, p.27).

É o estádio em que se encontra o tumor que determina se o câncer pode ser removido cirurgicamente. A União Internacional Contra o Câncer (UICC) criou uma classificação, denominada estadiamento para definir se um tumor está em estado avançado ou não. Essa classificação tem como base três fatores: o tamanho do tumor, a avaliação da extensão dos linfonodos e a presença ou não de metástases.

Os casos são classificados em estádios que variam de 0 a IV graus crescentes de gravidade da doença (Diagnóstico..., 2009). O tratamento irá depen der diretamente do grau do estadiamento. Nesse sentido, se o tumor é diagnosticado em estádios mais avançados, os recursos terapêuticos se tornam mais limitados e mutilantes (Paci et al., 1996; Gerber et al., 1992, citado em Bergmann, 2000). Segundo Abreu e Koifman (2002, p.114), os estadiamentos III e IV chegam a corresponder a cerca de $60 \%$ dos diagnósticos iniciais. Um dos motivos para tal situação estaria no número insuficiente de mamógrafos.

Para o fisioterapeuta oncológico, é fundamental ter conhecimento do estádio em que se encontra o paciente. A má utilização dos recursos fisioterapêuticos poderá contribuir com a proliferação celular nas redes linfática e sanguínea. Assim como o tratamento para o câncer de mama, a cirurgia adequada varia de acordo com o tipo de tumor e o estágio da doença. Na cirurgia conservadora é retirada apenas uma parte da mama. Nas cirurgias radicais (mastectomia), a mama éretirada por completo e, eventualmente, éextraído também 
o músculo peitoral. Ambas as modalidades cirúrgicas são normalmente acompanhadas pela retirada de nódulos linfáticos das axilas (linfonodos). A mastectomia é um procedimento cirúrgico que evita a disseminação do câncer. ${ }^{22}$

As cirurgias acarretam, como foi dito, danos físicos importantes para o paciente. A imensa maioria submete-se a abordagem cirúrgica da axila, e o pós-operatório pode levar a complicações como seroma, deiscência da ferida cirúrgica, aderência cicatricial, retrações, fibroses, dor na incisão cirúrgica, dor na região cervical, alterações de sensi bilidade, alterações posturais, diminuição da capacidade respiratória, diminuição de amplitude de movimento, fraqueza, encurtamentos musculares e linfedema precoce. Entre todas as complicações decorrentes do tratamento para câncer de mama, o linfedema secundário do membro superior, éa mais frequente (Leitch et al., 1998; Pezner et al., 1986; Clarysse, 1993; Segerstron, 1992; Tadych, Donegan, 1987, citado em Bergmann, 2000).

\section{A fisioterapia precoce e o pós-operatório}

A fisioterapia precoce tem como objetivo prevenir complicações que poderão ocorrer caso não se tenha o adequado conhecimento dos fatores prognósticos. Do ponto de vista clínico, os mais importantes são o tamanho do tumor eo comprometimento axilar. Segundo Abreu e Koifman (2002, p.115), o conhecimento dos fatores prognósticos é fundamental na determinação dos programas terapêuticos e fisioterapêuticos.

A prevenção de complicações deve estar presente em todas as fases do câncer de mama: no diagnóstico; no tratamento (quimioterapia, radioterapia, hormonioterapia e cirurgia); na recorrência da doença e nos cuidados paliativos. Segundo Batiston e Santiago (2005, p.30-31), é fundamental iniciar um programa fisioterapêutico precocemente, quando as pacientes ainda não apresentam complicações, como limitações de movimentos, dor, linfedema e aderência cicatricial. No entanto, muitas são encaminhadas tardiamente, o que diminui a probabilidade de recuperação.

No pós-operatório imediato, a intervenção fisioterapêutica busca "identificar alterações neurológicas ocorridas durante o ato cirúrgico, presença de sintomas ál gicos, edema linfático precocee alterações na dinâmica respiratória" (Brasil, 2004, p.84). No decorrer do tratamento, objetiva-se a adequada recuperação funcional e, consequentemente, melhor qualidade de vida para a paciente (Guia..., s.d.). Alguns trabalhos têm chamado atenção para a importância da avaliação da qualidade de vida em pacientes submetidos à mastectomia como uma estratégia de intervenção terapêutica. A oncologia foi, segundo Makluf, Dias e Barra (2006, p.50), a especialidade que se viu confrontada com as necessidades de se avaliar qualidade de vida.

Hoje, duas situações predominam no tratamento do câncer: de um lado, a cura completa, sem sequelas físicas e/ou funcionais; de outro, o que se observa é a necessidade de um tratamento mais agressivo que pode deixar limitações significativas. Neste segundo caso, se faz necessário o envolvimento ativo de uma equipe multidisciplinar, composta por médicos, enfermeiros, psicólogos, fisioterapeutas, terapeutas ocupacionais, assistentes sociais e nutricionistas. Mas é importante que a equipe de saúde esteja preparada para diagnosticar e intervir precocemente. 
Segundo Rezende et al. (2006, p.37-38), considerando a expectativa de sobrevida para mulheres submetidas à mastectomia radical, tornou-se fundamental o aprimoramento das técnicas de reabilitação para proporcionar uma adequada recuperação física e mental. Nos últimos anos, muitas propostas de tratamento foram desenvolvidas com o intuito de minimizar as complicações pós-operatórias. ${ }^{23}$ Várias são as terapias indicadas, algumas baseadas em exercícios de alongamento global e fortalecimento muscular, outras em exercícios ativos assistidos progredindo para exercícios ativos resistidos, exercícios respiratórios, manobras de drenagem linfática manual, além de movimentos de facilitação neuromuscular proprioceptiva e atividades funcionais. Pode-se ainda aplicar técnicas e condutas fisioterapêuticas para melhora da postura, como reeducação postural gl obal (RPG), antiginástica, cinesioterapia. Os recursos anal gésicos (Tens, crioterapia, mobilização passiva, técnicas de relaxamento muscular) complementam o tratamento fisioterapêutico (Bergmann et al., 2006, p.100).

A intervenção da fisioterapia pode ser medida pelo grau de independência alcançado pela paciente, proporcionando alívio da dor, diminuindo os riscos de infecção, aumentando a mobilidade de membros superiores e reduzindo a necessidade de medicamento como anal gésicos.

Durante o período de internação o enfoque é global, prevenindo, minimizando e tra tando complicações respiratórias, motoras e circulatórias. A dor é uma das principais e mais frequentes queixas da paciente, devendo ser valorizada, controlada e tratada em todas as etapas da doença. Segundo Bergmann et al. (2006, p.100-101), as diversas técnicas para analgesia caracterizam a fisioterapia em oncologia (Bergmann, 2008).

\section{O estabelecimento da profissão e a consolidação da fisioterapia em oncologia}

A atuação abrangente e a acumulação de experiência profissional estão redirecionando o campo de atenção do fisioterapeuta. Esse profissional vem atuando em todos os níveis de assistência à saúde, incluindo a prevenção, a promoção, o tratamento e a recuperação, com ênfase no movimento e na função.

Embora o processo de profissionalização da fisioterapia no Brasil tenha sido marcado pela hegemonia médica na saúde, assim como as demais profissões em saúde, é possível dizer que a profissão vem crescen do no domínio do conhecimento e da atuação profissional. O fisioterapeuta, que até bem pouco tempo era visto como um auxiliar do médico, hoje é reconhecido, pela legislação em vigor, como um profissional capaz para avaliar e tratar o seu paciente de forma autônoma, com conhecimento suficiente para múltiplas funções na atenção à saúde.

O fisioterapeuta vem adquirindo prestígio profissional, cada vez mais reconhecido como um ator importante nos serviços de saúde. Seu espaço diante da autoridade médica, ainda que reduzido, tem reconhecimento legítimo. Isso se deve, em parte, a ter conseguido reivindicar algum monopólio sobre uma área do conhecimento - a cinesioterapia funcional - e a sua atuação em ambulatórios, consultórios, centros de reabilitação, Programa Saúde da Família e nas atividades de ensino, tanto na graduação como nos programas de pósgraduação. 
O que determina a autonomia e o campo de atuação de uma profissão? O conhecimento, a capacidade científica e a credibilidade. A consolidação de uma tradição de pesquisa dependerá do grau de comprometimento profissional, consenso e adesão da comunidade. Tais el ementos são prérequisitos para a continuação de uma tradição de pesquisa. Somente o profissional comprometido conseguirá desenvolver estruturas cognitivas estáveis para lidar com os problemas que comprometem diretamente a qualidade do trabal ho científico e que afetam a estabilidade e a identidade da comunidade (Kuhn, 1989, p.30-31).

Wilensky (1964, p.137-142) enfatiza que o grau deautonomia de uma profissão é medido não só pela competência, habilidade e jurisdição a padrões de treinamento, mas também pela aderência ao ideal de serviço e às normas de conduta profissional. Um profissional deve, ainda, convencer o público de que seus serviços são confiáveis. Freidson $(1975,1998)$ lembra que a autonomia é condição para alcançar-se legitimidade e mérito na atividade científica. O reconhecimento de uma profissão está intimamente relacionado ao prestígio de seus profissionais. Assim como o médico necessita conquistar a confiança de seus clientes - mediante a resolução de problemas que os afligem -, o fisioterapeuta precisa mostrar capacidade de controlar uma área de atividade, ser possuidor de conhecimento técnico e especializado na 'arte e técnica' do cuidar.

A fisioterapia, reconhecida e habilitada para a prevenção de doenças e recuperação da saúde, vem formando profissionais capazes de controlar uma área de atividade e preocupados com a realidade social. As discussões em torno da formação e da atuação surgem com a necessidade de estudar e trabalhar a saúde como uma condição social de múltiplas dimensões. As universidades, em especial as públicas, vêm propondo cada vez mais alternativas de atuação socialmente relevantes. A saúde coletiva tem contribuído nesse sentido, buscando a articulação da área da saúde com outras áreas de conhecimento, como por exemplo a das ciências sociais. Essa aproximação tem permitido ao profissional de saúde pensar e agir relacionando-se com as diversas áreas de conhecimento.

A fisioterapia vem experimentando um crescimento, nas duas últimas décadas, em especial em oncologia, e a preocupação quanto à formação tem acompanhado esse crescimento. Pesquisadores, coordenadores e professores de vários estados brasileiros estão conscientes da necessidade de mudanças nos currículos, para garantir a formação de um profissional competente e que contribua para a construção do setor saúde no Brasil, proporcionando mel hores condições de vida, integração psicossocial do indivíduo e condições para o exercício da cidadania. Essa atitude é reforçada por Deliberato (2002, p.6), quando afirma que "o caminho do profissional fisioterapeuta na direção das ações preventivas, em vez de centralizarse no modelo curativo, representa na verdade uma via inteligente e exequível".

Por fim, cabe destacar que, uma vez que a saúde passou a ser associada às condições sociais, e não mais simplesmente vinculada ao tratamento das doenças, a fisioterapia precoce passou a desempenhar um papel fundamental. No tocante à fisioterapia em oncologia, busca-se levar uma melhor qualidade de vida aos pacientes com câncer, minimizando os efeitos adversos do tratamento. Os resultados positivos estão relacionados à recuperação físico-funcional. Eles advêm da aplicação sistematizada de recursos terapêuticos diversos, com o foco sempre voltado para o controle dos sintomas imediatos referidos pelo paciente (Bergmann, 2008). 


\section{Considerações finais}

Cada vez mais se faz necessária a atuação da fisioterapia em pacientes com câncer de mama, do ponto de vista do tratamento e da prevenção, para minimizar os efeitos da cirurgia radical ou mesmo superá-los. O carcinoma mamário é o segundo tipo de câncer mais frequente no mundo e o mais comum entre as mulheres brasileiras. A fisioterapia expandiu e consolidou sua atuação em oncologia, em especial no tratamento do câncer de mama feminino. A preocupação com a formação profissional tem acompanhado esse crescimento. O treinamento e a competência em fisioterapia são fundamentais para se alcançarem os resultados esperados. O fisioterapeuta precisa ter claro o objetivo de tratamento, facilitando a aceitação da paciente ao atendimento. É preciso considerar o caráter preventivo e antecipar possíveis complicações para evitar sofrimentos desnecessários. O profissional bem formado estará apto a prever complicações. O benefício a ser buscado é a preservação da vida e o alívio dos sintomas, dando oportunidade, sempre que possível, para a independência funcional da paciente. A reinserção em suas atividades de vida diária é importante para restaurar a autoestima. "A ausência de autoestima faz com que sejamos algozes de nós mesmos", disse um médico de renome. Erving Goffman (1985) o cientista social estudioso das 'identidades deterioradas', não diria outra coisa. Cumpre respeitar, resguardar e resgatar identidades perdidas. Ao contribuir efetivamente para a retomada da atividade física, a fisioterapia pode colaborar para a conquista da autoestima pela paciente, para a aceitação de si própria, de seus limites e possibilidades funcionais.

\section{AGRADECIMENTOS}

A autora agradece as sugestões feitas por Gulnar Azevedo e Silva M endonça a uma versão anterior deste artigo.

\section{NOTAS}

${ }^{1}$ Nesta e nas demais citações de textos em outros idiomas, a tradução é livre.

${ }^{2}$ Conforme as Diretrizes Curriculares Nacionais do Curso de Fisioterapia (Brasil, 4 mar. 2002).

${ }^{3}$ A esse respeito, ver http://www.inca.gov.br/index.asp (acesso em 12 de julho de 2008).

${ }^{4}$ Berta e Karel Bobath criaram o método Bobath ou conceito neuroevolutivo. O método foi criado nos anos 1950 para tratamento de bebês, crianças e adultos com lesões no sistema nervoso. É uma técnica de reabilitação neuromuscular que utiliza os reflexos e os estímulos sensitivos para inibir ou provocar uma resposta motora. Ver Davies, 1996.

${ }^{5} \mathrm{Na}$ década de 1960 eram 15 mil fisioterapeutas em atividade em todo o país (Barros, 2003).

${ }^{6}$ M édico da Santa Casa de Misericórdia de São Paulo, que obteve o primeiro sucesso na extirpação total de um estômago atacado por um câncer do piloro. Foi fundador e primeiro diretor da Faculdade de Medicina e Cirurgia de São Paulo, até 1920 (Marinho, 2003).

${ }^{7}$ Os primeiros aparel hos de raios $X$ chegaram ao Brasil em final da década de 1890, trazidos por médicos do Rio de Janeiro e da Bahia que retornavam de viagens à Europa. No Rio de Janeiro, o médico Álvaro Alvim foi o pioneiro no uso desses equipamentos. Em 1897 instalou, em seu consultório de fisioterapia, um aparel ho de raio $X$ e trabal hou com a técnica até 1928 , quando faleceu em virtude de lesões ocasionadas pelo seu uso (Teixeira, Fonseca, 2007, p.23).

${ }^{8} \mathrm{O}$ curso tinha dois anos de duração e funcionou até 1958.

${ }^{9}$ Através da portaria G.R. 347 de 7 de abril de 1967; ver Barros, 2003. 
${ }^{10}$ Em Minas Gerais, o primeiro curso de fisioterapia foi criado em virtude da necessidade de mão de obra especializada para atendimento de portadores de deficiências físicas nos Hospitais Arapiara e da Baleia. Os cursos de fisioterapia e fisiatria eram lecionados na Faculdade de Ciências Médicas de Minas Gerais (FCM M G). Esses cursos foram organizados por médicos mineiros (ortopedistas e fisiatras) que trouxeram para o país um modelo norte-americano de reabilitação, uma vez que se especializaram em ortopedia ou fisiatria nos Estados Unidos, alguns no Instituto de Reabilitação da Universidade de Nova York. Ver Nascimento, 2004; Nascimento et al., 2006.

${ }^{11}$ A partir dos anos 1960, é significativa a participação dos fisioterapeutas na elaboração dos currículos, buscando adequá-los às suas necessidades profissionais, rompendo, de certa forma, com a submissão ao médico (Oliveira, 2004, p.253).

12 Por outro lado, segundo Oliveira (2004, p.259), os médicos criticavam o fato de os fisioterapeutas reclamarem para si atividades que eram próprias dos fisiatras, especialistas em medicina física e reabilitação. A Sociedade Brasileira de M edicina Física e Reabilitação de São Paulo se posicionou contra a intromissão 'abusada' nas atividades profissionais que 'sempre' foram atribuições exclusivas dos fisiatras.

${ }^{13}$ Através do parecer 388/63, homologado pela portaria 511/64 do MEC; ver Rebelatto, Botomé, 1999, p.65.

${ }^{14}$ Desde então foram realizados 17 congressos de fisioterapia em várias partes do país.

15 Segundo Barros (2003), a criação desses conselhos se deve à implantação, meses antes, do Plano de Classificação de Cargos no serviço público federal, o qual, na tentativa de reduzir cargos e carreiras, engl obava diferentes profissionais com a mesma denominação. Foi por esse motivo que os fisioterapeutas, terapeutas ocupacionais e fonoaudiólogos ficaram sob a denominação de técnicos de reabilitação, que perdurou até 1984.

${ }^{16}$ Coutinho e Soares (2000) colocam em discussão as premissas de que as decisões na área de saúde pública seguem considerações epidemiológicas racionais, baseadas em índices de risco associados ao contágio, a taxas de incidência e de mortalidade etc. Ainda que tais fatores venham a nortear os investimentos em programas de controle e prevenção, os autores baseiam-se em estatísticas norteamericanas para demonstrar que o acesso às verbas federais está intimamente relacionado à capacidade de negociação dos grupos organizados. Os autores citam os exemplos das políticas de câncer da próstata e de mama, nos Estados Unidos. Embora as taxas de mortalidade sejam muito próximas para ambas as doenças, as campanhas de câncer de mama recebem naquele país mais recursos, em função da "eficácia das ações dos grupos de pressão na construção de um consenso político em torno da relevância de suas causas" (p.33).

${ }^{17}$ No Brasil, o carcinoma mamário é o que mais causa mortes entre as mulheres. Os índices são maiores que em outros tipos de câncer (Brasil, s.d. - Inca, Relatório anual 2007). As estimativas para os anos de 2008 e 2009 são desoladoras. Cerca de 49 mil mulheres terão câncer de mama. Os estados com os maiores índices são: São Paulo (15.640), Rio de Janeiro (7.680), Rio Grande do Sul (4.880), Minas Gerais (4.280) e Santa Catarina (1.610) (Brasil, 2008).

${ }^{18}$ A esse respeito ver http://www1.inca.gov.br/conteudo view.asp?id=682 (acesso em 28 de julho de 2008). O Hospital do Câncer III possui um serviço de fisioterapia, unidade responsável pelo atendimento exclusivo de pacientes com câncer da mama do Instituto Nacional de Câncer.

${ }^{19}$ Ver http://www1.inca.gov.br/conteudo_view.asp?id=682 (acesso em 28 de julho de 2008).

${ }^{20}$ Um estudo importante publicado no Sao Paulo Medical Journal chama atenção para a relação positiva entre perda de peso corporal e câncer de mama (Vasconcelos, Mendonça, Sichieri, 2001).

${ }^{21}$ Ver, a respeito, o site do Instituto Nacional do Câncer (http://www.inca.gov.br/conteudo_view.asp; acesso em 1 de julho de 2008).

22 Ver, a respeito, o site Oncoguia (http://www.oncoguia.com.br/site/interna.php; acesso em 28 de julho de 2008) e Guia..., s.d. (http://cancermama.net/; acesso em 2 de junho de 2008).

${ }^{23}$ Ver descrição das rotinas do Serviço de Fisioterapia do Hospital do Cancer III/Inca em Bergmann et al., 2006. 


\section{REFERÊNCIAS}

ABREU, Evaldo; KOIFMAN, Sérgio. Fatores prognósticos do câncer da mama feminina. Revista Brasileira de Cancerologia, Rio de Janeiro, v.48, n.1, p.113-31. 2002.

ASOCIACIÓN MÉDICA AMERICANA. Estado actual de la fisioterapia. Boletín de la Oficina Sanitaria Panamericana, Washington, v.16, n.4, p.316-22. 1937.

BARROS, Fábio Batalha Monteiro.

Poliomielite, filantropia e fisioterapia: o nascimento da profissão de fisioterapeuta no Rio de Janeiro dos anos 1950. Revista Ciência e Saúde Coletiva, Rio de Janeiro, v.13, n.3, p.941-954. 2008.

BARROS, Fábio Batalha Monteiro.

Autonomia profissional do fisioterapeuta ao longo da história. Revista FisioBrasil, Rio de Janeiro, n.59, p.20-31. 2003.

BATISTA, Hilton.

A história da medicina de reabilitação. Rio de Janeiro. Disponível em: http://www.abbr.org.br/ historiadamedicina.php. Acesso em: 12 jul. 2008. 1988.

BATISTON, Adriane Pires; SANTIAGO, Silvia

Maria.

Fisioterapia e complicações físico-funcionais após tratamento cirúrgico do câncer de mama. Fisioterapia e Pesquisa, São Paulo, v.12, n.3, p.30-35. 2005.

BERGMANN, Anke.

Fisioterapia no câncer de mama: assistência, ensino e pesquisa. Trabal ho apresentado na Semana de Fisioterapia da Universidade Estácio de Sá, maio de 2008. Petrópolis. 2008.

BERGMANN, Anke.

Prevalência de linfedema subsequente a tratamento cirúrgico para câncer de mama no Rio de Janeiro. Dissertação (Mestrado) - Programa de Pós-graduação em Saúde Pública, Escola Nacional de Saúde Pública, Fundação Oswaldo Cruz, Rio de Janeiro. 2000.

BERGMANN, Anke et al.

Fisioterapia em mastologia oncológica: rotinas do Hospital do Câncer III/INCA. Revista Brasileira de Cancerologia, Rio de Janeiro, v.52, n.1, p.97-109. 2006.

BERGMANN, Anke et al.

Diagnóstico do linfedema: análise dos métodos empregados na avaliação do membro superior após linfadenectomia axilar para tratamento do câncer de mama. Revista Brasileira de Cancerologia, Rio de Janeiro, v.50, n.4, p.311-320. 2004.
BERGMANN, Anke et al.

Morbidade após o tratamento de câncer de mama. Fisioterapia Brasil, Rio de Janeiro, v.1, n.2, p.101-108. Disponível em: http:// educacaofisicarj.tripod.comfisioterapiabrasil. pdf \#page $=52$. Acesso em: 2 jun. 2008. 2000.

BRASIL.

Ministério da Saúde. Secretaria de Atenção à Saúde. Instituto Nacional do Câncer.

Coordenação de Prevenção e Vigilância de Câncer. Estimativas/2008: incidência de câncer no Brasil. Rio de Janeiro: Inca. 2008.

BRASIL.

Ministério da Saúde. Instituto Nacional do Câncer. Controle do câncer de mama: documento de consenso. Rio de Janeiro: Inca. 2004.

BRASIL.

Consel ho Nacional de Educação. Câmara de Educação Superior. Resolução CNE/CES 4 de 19 de fevereiro de 2002. Diário Oficial da União, Brasília, seção 1, p.11. 4 mar. 2002.

BRASIL.

Conselho Federal de Fisioterapia e Terapia Ocupacional. Resolução Coffito n.80. Diário Oficial da União, Brasília, n.93, seção I, p.7609. Baixa atos complementares à Resolução Coffito 8 - exercício profissional do fisioterapeuta. 21 maio 1987.

BRASIL.

Decreto-lei n.938. Provê sobre as profissões de fisioterapeuta e terapeuta ocupacional, e dá outras providências. Diário Oficial da União, Brasília. 13 out. 1969.

BRASIL.

Decreto-lei n.57.363. Concede reconhecimento à Escola de Reabilitação do Rio de Janeiro da Associação Brasileira Beneficente de Reabilitação. Diário Oficial da União, Brasília. 2 dez. 1965.

BRASIL.

Ministério da Saúde. Instituto Nacional do Câncer. Câncer de mama: a cura é possível, conhecer é necessário. Folheto. Disponível em: http://www.inca.gov.br/publicacoes/ Folheto_mama.pdf. Acesso em: 20 maio 2008. s.d.

\section{BRASIL.}

Ministério da Saúde. Instituto nacional do Câncer. Relatório anual 2007. Rio de Janeiro: Inca. Disponível em: http://www1.inca.gov.br/ inca/Arquivos/Relatorios/inca_relatorio2007_ web.pdf. Acesso em: 20 maio 2008. s.d. 
BRASIL.

Ministério da Saúde. Instituto nacional do Câncer. Relatório anual 2005. Rio de Janeiro: Inca. Disponível em: http://www1.inca.gov.br/ inca/Arquivos/RelatorioGestao/

RELATGESTAO2005.pdf. Acesso em: 12 maio 2008. s.d.

\section{BRASIL.}

Ministério da Saúde. Instituto Nacional do Câncer. Relatório anual 2004. Rio de Janeiro: Inca. Disponível em: http://wwwl.inca.gov.br/ conteudo view.asp?id=1724. Acesso em: 12 maio 2008. s.d.

CARRARA, Sérgio.

Tributo a Vênus: a luta contra a sífilis no Brasil, da passagem do século aos anos 40. Rio de Janeiro: Editora Fiocruz. 1996.

CASTRO SANTOS, Luiz A.

A duras penas: estratégias, conquistas e desafios da Enfermagem em escala mundial. História, Ciências, Saúde - Manguinhos, Rio de Janeiro, v.15, n.1, p.13-28. 2008.

CASTRO SANTOS, Luiz A., FARIA, Lina. As ocupações supostamente subalternas: o exemplo da enfermagem brasileira. Saúde e Sociedade, São Paulo, v.17, n.2, p.35-44. 2008.

CASTRO SANTOS, Luiz A; FARIA, Lina. A cooperação internacional e a enfermagem de saúde pública no Rio de Janeiro e São Paulo. Horizontes, Bragança Paulista, v.22, n.2, p.123-148. 2005.

\section{CLARYSSE, A}

Lymphoedema following breast cancer treatment. Acta Clinica Belgica Supplementum, Brussels, n.15, p.47-50. 1993.

COUTINHO, Marília; SOARES, Gláucio A. Dillon.

Homens: a maioria desorganizada. Dados, Rio de Janeiro, v.43, n.2. Disponível em: http:// www. scielo.br/scielo.php?pid=s0011-

$52582000000200004 \&$ script=sci_arttext\&tlng=in . Acesso em: 10 maio 2010.

DELIBERATO, Paulo CP.

Fisioterapia preventiva: fundamentos e aplicações. São Paulo: Manole. 2002.

DIAGNÓSTICO...

Diagnóstico e estadiamento. Disponível em: http://www.oncoguia.com.br/site/ interna. php?cat $=113 \& i d=1231 \&$ menu $=2$. Acesso em: 20 maio 2010. 2009.

FARIA, Lina.

Educadoras sanitárias e enfermeiras de saúde pública: identidades profissionais em construção. Cadernos Pagu, Campinas, n.27, p.173-212. 2006
FIGUEIRÔA, Regina Maria.

Aspectos da evolução histórica do fisioterapeuta no Brasil em especial no Rio de Janeiro.

(Monografia) - Instituto Brasileiro de Medicina de Reabilitação, Rio de Janeiro. 1996.

FREIDSON, Eliot.

Renascimento do profissionalismo. São Paulo: EdUSP. 1998.

FREIDSON, Eliot.

Profession of medicine: a study of the sociology of applied knowledge. New York: Dodd, Mead \& Company. 1975.

GALVÃO, Aires M.F.

Brasil, a fisioterapia e o tempo. Fisiobrasil, Rio de Janeiro, n.38, p.22-26. 2000.

GERBER, L. et al.

Comparison of pain, motion, and edema after modified radical mastectomy vs. local excision with axillary dissection and radiation. Breast Cancer Research and Treatment, Dordrecth, n.21, p.139-145. 1992.

GOFFMAN, Erving.

A representação do eu na vida cotidiana. Petrópolis: Vozes. 1985.

GUIA...

Guia câncer de mama. Disponível em: http:// www.cancermama.net/. Acesso em: 2 jun. 2008. s.d.

GUIMARÃES, Darcy da Silva et al.

A história da mastologia no Inca. Revista Brasileira de Cancerologia, Rio de Janeiro, v.48, n.1, p.135-138. 2002.

KISNER, Carolyn; COLBY, Lynn Allen. Exercícios terapêuticos: fundamentos e técnicas. 4.ed. São Paulo: Manole. 2005

KUHN, Thomas S.

A estrutura das revoluções científicas. 3.ed. São Paulo: Perspectiva. (Debates - Ciência). 1989.

LEITCH, A. Marilyn et al.

American Cancer Society Lymphedema Workshop. Workgroup I: Treatment of the axilla with surgery and radiation - preoperative and postoperative risk assessment. Cancer Supplement, Bethesda, v.83, n.12, p.2877- 2879. 1998.

MAKLUF, Ana Silvia Diniz; DIAS, Rosângela C; BARRA, Alexandre A.

Avaliação da qualidade de vida em mulheres com câncer de mama. Revista Brasileira de Cancerologia, Rio de Janeiro, v.52, n.1, p.49-58. 2006.

MARINHO, Maria Gabriela.

Elites em negociação: breve história dos acordos entre a Fundação Rockefeller e a Faculdade de 
Medicina de São Paulo (1916-1931). Bragança Paulista: EdUSF. 2003.

MARQUES, Amélia P.; SANCHEZ, Eugênio Lopes. Origem e evolução da fisioterapia: aspectos históricos e legais. Revista de Fisioterapia da Universidade de São Paulo, São Paulo, v.1, n.1, p.5-10. 1994.

MIRRA, Antonio Pedro.

Registros de câncer no Brasil e sua história. São Paulo: Ministério da Saúde; Inca. 2005.

NASCIMENTO, Maria do Carmo.

A profissão de fisioterapia em Minas Gerais: uma história oral de suas raízes. Dissertação (Mestrado) - Programa de Pós-graduação em Ciências da Reabilitação, Escola de Educação Física, Fisioterapia e Terapia Ocupacional, Universidade Federal de Minas Gerais, Belo Horizonte. 2004.

NASCIMENTO, Maria do Carmo et al. A profissionalização da fisioterapia em Minas Gerais. Revista Brasileira de Fisioterapia, São Carlos, v.10, n.2, p.241-247. 2006.

OLIVEIRA, Valéria Rodrigues Costa de. Reconstruindo a história da fisioterapia no mundo. Revista Estudos, São Paulo, v.32, n.4, p.509-534. 2005.

OLIVEIRA, Valéria Rodrigues Costa de. A construção da identidade profissional do fisioterapeuta. Revista Estudos, São Paulo, v.31, n.2, p.247-274. 2004.

OPAS.

Organización Panamericana de la Salud. Scientific institutions in Latin America: municipal institute of radiology and physiotherapy. Boletín de la Oficina Sanitaria Panamericana, Washington, v.20, n.2, p.153-56. 1941.

$\mathrm{PACl}$, E. et al.

Long-term sequelae of breast cancer surgery. Tumori, Milano, n.82, p.321-324. 1996.

PEZNER, R. et al.

Arm lymphedema in patients treated conservatively for breast cancer: relationship to patient age and axillary node dissection technique. International Journal of Radiation Oncology, Biology and Physics, Elmsford, n.12, p.2079-2083. 1986.

POLLACK, Susan.

Breast cancer: piecing together the puzzle. In Breast Cancer. Searching for answers. Harvard Public Health Review, Boston, v.3, n.1, p.4-10. 1992.

REBELATTO, José Rubens; BOTOMÉ, Sílvio Paulo.

Fisioterapia no Brasil: fundamentos para uma ação preventiva. São Paulo: Manole. 1999.
REIS, Mariana C.S.

A formação do fisioterapeuta numa instituição pioneira: a Escola de Reabilitação do Rio de Janeiro (1956-1976). Dissertação (M estrado) Universidade Católica de Petrópolis, Petrópolis. 2004.

REZENDE, Laura Ferreira de et al.

Exercícios livres versus direcionados nas complicações pós-operatórias de câncer de mama. Revista da Associação M édica Brasileira, São Paulo, v.52, n.1, p.37-42. 2006.

SALM ÓRIA, Jordana Gargioni; CAMARGO, Wander Amaral.

Uma aproximação dos signos: fisioterapia e saúde, aos aspectos humanos e sociais. Saúde e Sociedade, São Paulo, v.17, n.1, p.73-84. 2008.

SANCHEZ, Eugênio Lopes.

Histórico da fisioterapia no Brasil e no Mundo. Atualização Brasileira de Fisioterapia, São Paulo, v.1, n.3, p.6-11. 1984.

SANGLARD, Gisele.

Entre os salões e o laboratório: Guilherme Guinle, a saúde e a ciência no Rio de Janeiro,

1920-1940. Rio de Janeiro: Editora Fiocruz. 2008.

SODRÉ, Antonio Augusto Azevedo.

Freqüência do câncer no Brasil. Brasil M édico, Rio de Janeiro, v.18, n.23, p.229-232. 1904.

SANTOS FILHO, Lycurgo de Castro.

História geral da medicina brasileira. São Paulo: Hucitec; EdUSP. 1991.

SANTOS FILHO, Lycurgo de Castro. Pequena história da medicina brasileira. São Paulo: Parma. 1980.

SCHWARTZMAN, Simon.

Formação da comunidade científica no Brasil. São Paulo: Editora Nacional. 1979.

SEGERSTRON, B. et al.

Factors that influence the incidence of brachial edema after treatment of breast cancer.

Scandinavian Journal of Plastic and Reconstructive Surgery and Hand, Stockholm, n.26, p.223-227. 1992.

TADYCH, K.; DONEGAN, W.

Post mastectomy seromas and wound drainage. Surgery, Gynecology \& Obstetrics, Chicago, n.165, p.483-487. 1987.

TEIXEIRA, Luiz A., FONSECA, Cristina M.O. De doença desconhecida a problema de saúde pública: o INCA e o controle do câncer no Brasil. Rio de Janeiro: Ministério da Saúde. 2007.

USP.

Universidade de São Paulo. Hospital das Clínicas. Faculdade de Medicina. História do 
HC: os outros institutos. Disponível em: http:// www.hcnet.usp.br/historiahc/institutos.htm. Acesso em: 12 jul. 2008. 1997.

VASCONCELOS, Anelise Bezerra de; MENDONÇA, Gulnar Azevedo e Silva; SICHIERI, Rosely.

Height, weight, weight change and risk of breast cancer in Rio de Janeiro, Brazil. São Paulo M edical Journal, São Paulo, v.119, n.2, p.62-66. 2001.

\section{WILENSKY, Harold L.}

The professionalization of everyone? American Journal of Sociology, Chicago, n.70, p.137-158. 1964.

\section{uUuUUU}

